\title{
Nahrungsmittelunverträglichkeit und Nahrungsmittelallergie
}

\section{Was darf Ihr Patient noch essen?}

\author{
Nahrungsmittelallergien sind - wie andere Allergien auch - auf dem Vor- \\ marsch. Die Diagnose basiert primär auf einer detaillierten Anamnese und \\ dem Führen eines Ernährungstagebuchs. Zur Diagnosesicherung sind dann \\ zusätzlich klinische und laborchemische Tests erforderlich.
}

— Nicht jede Nahrungsmittelunverträglichkeit ist einer Allergie. „Bevor eine allergische Ursache diskutiert wird, müssen andere Möglichkeiten wie eine toxische Ursache oder eine Maldigestion wie etwa eine Laktose- oder Fruktoseintoleranz ausgeschlossen sein“, sagte Prof. Elke Roeb von der Gastroentrologischen Klinik der Universität in Gießen. Von Nahrungsmittelallergien sind heute ca. $6 \%$ der Kinder und 3-4\% der erwachsenen Bevölkerung betroffen.

\section{Von leichter Übelkeit bis zum anaphylaktischen Schock}

Nahrungsmittelallergien können sich an verschiedenen Organen wie Haut, Respirationstrakt, Verdauungstrakt oder Kreislaufsystem manifestieren. Die Symptomatik kann wenige Minuten nach der Nahrungsaufnahme, aber auch erst Stunden danach auftreten. „Bei einer Nahrungsmittelallergie am Gastrointesti-

\section{Tabelle 1}

Diagnostik bei V. a. Nahrungsmittelallergie

_ Bestimmung des Gesamt-IgE im Serum

- Bestimmung von allergenspezifischen lgE-Antikörpern (z. B. RastTest)

- Bestimmung von allergenspezifischen lgE-Antikörpern in verschiedenen Abschnitten des Verdauungstraktes durch gastrointestinale Lavage oder Biopsien

_ Hauttest mit verschiedenen Nahrungsmittelextrakten (z. B. Prick-Test) - Bestimmung von Methylhistamin im 24-h-Urin während der üblichen Ernährung und im Rahmen einer Ausschlussdiät (Kartoffel-Reis-Diät) naltrakt sind die Symptome relativ unspezifisch“, so Roeb. Sie umfassen Erbrechen, Übelkeit, abdominelle Schmerzen, Blähungen, Diarrhöen oder auch eine Obstipation. Außerhalb des Gastrointestinaltrakts können Hautrötungen, Bronchospasmen, Pruritus, Tränenfluss und Rhinitis auftreten. Die schwerste, lebensbedrohliche Form der Nahrungsmittelallergie ist der anaphylaktische Schock mit Blutdruckabfall, Tachykardie, Herzrhythmusstörungen und Bronchospastik.

Anamnese und Ernährungstagebuch Die Diagnose Nahrungsmittelallergie basiert auf einer detaillierten Anamnese inkl. Ernährungstagebuch und sorgfältiger klinischer Untersuchung. Zur Diagnosesicherung sind weitere Tests nötig. Dazu gehören der Prick-Test ebenso wie Ausschlussdiäten (Tab. 1) „Positive Testergebnisse dürfen nur bei assoziierten klinischen Symptomen als klinisch relevant interpretiert werden“, so Roeb.

Goldstandard bei der Diagnostik ist eine Ausschlussdiät und die anschließende erneute Exposition. „Sollte sich ein verdächtiges Lebensmittel oder ein Lebensmittelbestandteil herauskristallisieren, sollte eine mehrwöchige Ausschlussdiät erfolgen“, so Roeb. Anschließend wird im Rahmen einer kontrollierten Provokationsdiät der Beweis für eine Nahrungsmittelallergie angestrebt.

\section{Umstrittene unkonventionelle Maßnahmen}

In den letzten Jahren wurden unkonventionelle diagnostische Methoden immer beliebter. Diese beinhalten spezifische IgG-Antigene, Leukozytenantikörper und sublinguale oder intradermale Provokationstests, zytotoxische Nahrungs-

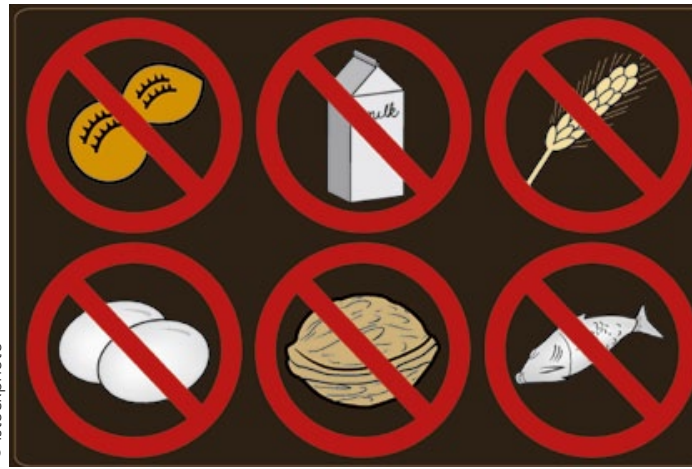

Nahrungsverbote nur nach sorgfältiger Diagnostik aussprechen!

mittel, kinesiologische und elektrodermale Testmethoden. „Zu diesen Methoden fehlen eine wissenschaftliche Rationale, eine Standardisierung und vor allem zuverlässige Nachweise der Reproduzierbarkeit“, so Roeb.

\section{Therapie je nach Manifestation}

Bei der Therapie der Nahrungsmittelallergien kommen nicht medikamentöse und medikamentöse Verfahren zum Einsatz. Ist die allergische Reaktion nur auf die Mukosa und Submukosa des MagenDarm-Traktes beschränkt, empfehlen sich lokal wirksame Therapieprinzipien wie Allergenkarenz, hypoallergene Kostformen und lokal wirksame Antiallergika. Spielt sich der allergische Immunprozess nicht nur lokal am Darm ab und treten IgE auch im vaskulären System auf, müssen zusätzlich systemisch wirksame Antihistaminika bzw. Glukokortikoide eingesetzt werden. Im Falle eines anaphylaktischen Schocks ist neben einer strikten Allergenkarenz auch eine antiallergische Dauertherapie erforderlich. Solche Patienten sollten mit einer Notfallmedikation mit Adrenalin-Autoinjektor, Antihistaminikum und einem Glukokortikoid zur Selbstmedikation ausgestattet werden.

Dr. med. Peter Stiefelhagen,

- Quelle: XIV. Gastroenterologie-Seminarwoche, 28.2.2012 in Titisee 